\title{
The Rotational Structure of the Hydrazoic Acid Bands in the Photographic Infra-Red
}

\author{
Eugene H. EysteR* \\ Department of Physics, University of Michigan, Ann Arbor, Michigan
}

(Received November 28, 1939)

\begin{abstract}
The third and fourth harmonics of the $\mathrm{N}-\mathrm{H}$ stretching frequency in hydrazoic acid, at 9547.31 and $12,412.19 \mathrm{~cm}^{-1}$, respectively, have been studied photographically under high dispersion. The rotational structure has been well resolved and completely analyzed, to yield the rotational constants in the ground and excited states. The effective moments of inertia in the ground state have been combined with other information to determine the structure of the molecule; the best values of the molecular parameters are:

$$
\begin{array}{ll}
\mathrm{N}_{1}-\mathrm{H}=1.012 \mathrm{~A} & \mathrm{~N}_{2}-\mathrm{N}_{3}=1.128 \mathrm{~A} \\
\mathrm{~N}_{1}-\mathrm{N}_{2}=1.241 \mathrm{~A} & \Delta H \mathrm{~N}_{1} \mathrm{~N}_{2}=110^{\circ} 52^{\prime} .
\end{array}
$$
\end{abstract}

$\mathrm{E}^{\mathrm{L}}$ LECTRON and $x$-ray diffraction experiments have fully confirmed the predictions of the modern structural theory concerning the structure of the azide group in ionic crystals and in covalent compounds. ${ }^{1}$ The infra-red spectra of covalent azides have not, however, been satisfactorily correlated with acceptable structures. It has been asserted that the rotational structure of the hydrazoic acid bands in the photographic infra-red demands the existence of two kinds of $\mathrm{HN}_{3}$ molecules, one of which is structurally inacceptable, and that the envelopes of methyl azide bands in the same region indicate that this molecule has a linear $\mathrm{C}-\mathrm{N}-\mathrm{N}-\mathrm{N}$ group, contrary to the electron diffraction results. ${ }^{2}$ It has consequently been thought useful to reinvestigate these spectra in order to determine whether or not these reported inconsistencies are real. The present paper presents the complete analysis of the rotational structure of the two strongest bands of hydrazoic acid in the photographic region, and demonstrates the fact that the resulting rotational constants determine a structure which is in complete harmony with theoretical predictions and with previous structure determinations in other covalent azides.

\section{EXPERIMENTAL}

The infra-red spectrum of hydrazoic acid was photographed by the author in the spectroscopic

* National Research Fellow in Chemistry.

1 The considerations leading to a prediction of the structures of the azide group in covalent and ionic compounds are summarized on page 185, L. Pauling, The Nature of the Chemical Bond (Cornell University Press, 1939). $2 \mathrm{~A}$ discussion of these results, with references to the original papers, is given in an interesting review article by Verleger, Physik. Zeits. 38, 83 (1937). laboratory of the Gates and Crellin Laboratories of Chemistry of the California Institute of Technology. The acid was prepared from recrystallized sodium azide (Eimer and Amend) by the method of Dennis and Isham. ${ }^{3}$ The gas was swept from the generator in a current of dry nitrogen, through a $50-\mathrm{cm}$ calcium chloride drying tube, and into a trap cooled in liquid air. This trap, attached to the generator by a Pyrex standard taper, was then transferred to a similar fitting on the six-meter Pyrex absorption tube, which was at once evacuated and sealed off while the explosive acid was still at liquid-air temperatures. The gas pressure was subsequently regulated by adjusting the temperature of the liquid acid in this trap. The whole of the absorption tube was shielded from stray radiation with black cloth, and a filter which cut out all wave-lengths shorter than $7000 \mathrm{~A}$ was interposed between the source and the tube to protect its contents from possible photochemical decomposition in the direct beam.

With the trap at room temperature, exploration photographs were taken with a Bausch and Lomb glass Littrow spectrograph set to record the region between 7500 and $12,000 \mathrm{~A}$ on Eastman I-Z plates. Four bands were observed, and the two stronger ones were then rephotographed in the first order of a twenty-one-foot concave grating spectrograph. For the band at 10,477A, the trap was held at $0^{\circ} \mathrm{C}$, and for the weaker $8058 \mathrm{~A}$ band it was held at $25^{\circ} \mathrm{C}$. Wave-length measurements on the prism spectrograms were

${ }^{3}$ L. M. Dennis and H. Isham, J. Am. Chem. Soc. 29, $216(1907)$. 


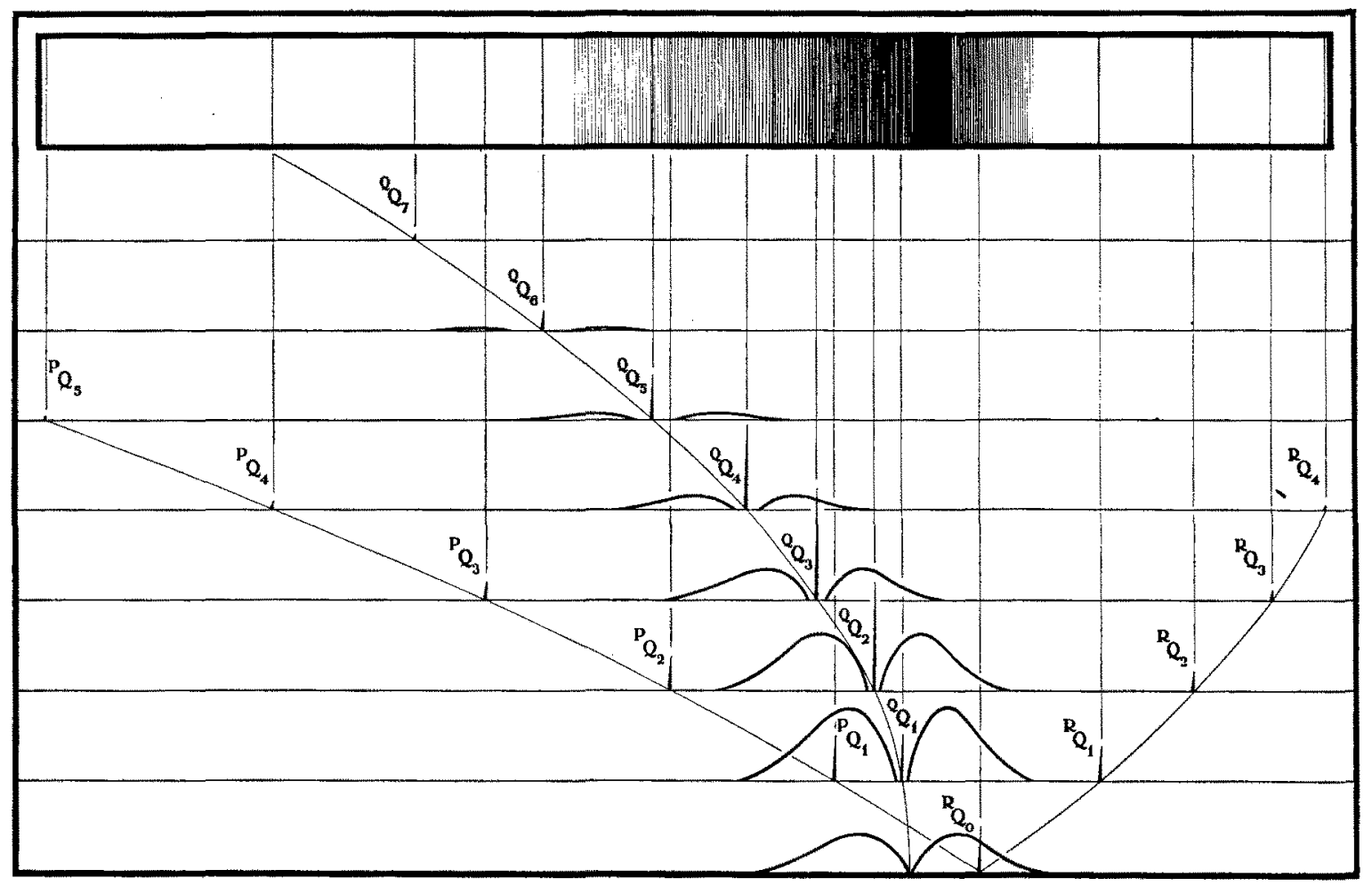

FIG. 1. The hydrazoic acid band at $9547 \mathrm{~cm}^{-1}$, and its analysis into constituent sub-bands and branches, Calculated intensities of the $K$-structure lines are indicated by their heights, and the intensities of the $J$-structure lines are given as envelopes.

made with respect to infra-red emission lines of copper, sodium, potassium, barium, and calcium, and on the grating spectrograms with respect to iron arc standards in the second and third orders. Eastman 144-R and I-Z plates, hypersensitized in ammonia, were used, and higher order spectra from the grating were removed by the filter already mentioned.

\section{Rotational Structure}

Since one may confidently expect the structures of hydrazoic acid and its methyl derivative to be analogous, the results of the electron diffraction study of methyl azide ${ }^{4}$ should provide a basis for the calculation of approximate moments of inertia for the acid itself. Such approximate moments enable one to estimate as probable values for Badger's rotational parameters $S$ and $\rho^{5}$ the following:

$$
S=-0.999 ; \quad \rho=+61.4 \text {. }
$$

${ }^{4}$ L. Pauling and L. O. Brockway, J. Am. Chem. Soc, 59 , 13 (1937).

5 R. M. Badger and L. R, Zumwalt, J. Chem. Phys, 6, $713(1938)$.
These values constitute a numerical statement of the fact that the rotational levels of hydrazoic acid should approximate those of a symmetric rotator of an extreme spindle shape. Hence it should be possible to treat the hydrazoic acid bands as symmetric rotator bands, except that lines receiving a large portion of their intensity from levels of high $J$ and low $K$ may show a splitting or fuzziness as the result of asymmetry:

The bands at $10,477 \mathrm{~A}$ and $8058 \mathrm{~A}$ undoubtedly correspond to the third and fourth harmonics of the $\mathrm{N}-\mathrm{H}$ stretching frequency, and both the third and fourth harmonic states of this vibration belong to the irreducible representation $A^{\prime}$ of the group $C_{s}$ of the planar hydrazoic acid. Molecular symmetry then does not require that these bands present rotational structures of pure types, and if we imagine the direction of electric moment change to be the $\mathrm{N}-\mathrm{H}$ bond direction, parallelperpendicular hybrid bands will in fact be anticipated. ${ }^{6}$

\footnotetext{
6 This molecule is so nearly symmetric that the type $B$ and $C$ bands may well be called perpendicular, and the
} 


\section{General features of observed bands}

These general expectations are well fulfilled by the observed bands. The third harmonic band is a hybrid of predominantly parallel structure, whose most striking feature is provided by the fact that the various parallel sub-bands, which usually coalesce to form a single parallel band, are here clearly separated as a result of the strong dependence of the small moment of inertia upon vibrational state; unusually marked convergence is correspondingly present in the perpendicular component. Fig. 1 shows a representation of the complete band, with its analysis into constituent branches. The coarse structure lines are separately represented and named, while only the envelopes of the closely spaced ${ }^{Q} R_{K}$ and ${ }^{Q} P_{K}$ branches are shown; though these latter are not specifically named, their naming is uniquely implied by the associated ${ }^{Q} Q_{K}$ branches. The nomenclature of lines will be evident from the following: the large letter describes the behavior of $J$ in the transition, the left-hand superscript describes the behavior of $K$, the right-hand subscript gives the value of $K^{\prime \prime}$, and the value of $J^{\prime \prime}$ follows the large letter in parentheses.

The fourth harmonic is a pure parallel band, and hence much simpler in appearance than the band of Fig. 1. As might be anticipated, the ${ }^{Q} Q$ branch sweeps back even more rapidly than in the third harmonic. Since the appearance of this band can be readily visualized from a consideration of the parallel component of the third harmonic band, it has not been separately illustrated. The frequencies and identifications of

TABLE I. The third $\mathrm{N}-\mathrm{H}$ harmonic band of hydrazoic acid at $9547.31 \mathrm{~cm}^{-1}$.

\begin{tabular}{|c|c|c|c|c|c|c|c|c|c|c|c|}
\hline$J$ & $Q_{P_{b}(J)}$ & $Q_{P_{4}(J)}$ & $Q_{P_{3}(J)}$ & $Q_{R_{\mathrm{a}}(J)}$ & $Q_{P_{2}(J)}$ & ${ }^{Q_{R_{1}}(J)}$ & $Q_{P_{1}(J)}$ & $K$ & ${ }^{P_{Q K}}$ & $Q_{Q K}$ & ${ }^{R} Q_{K}$ \\
\hline $\begin{array}{r}6 \\
7 \\
8 \\
9 \\
10 \\
11 \\
12 \\
13 \\
14 \\
15 \\
16 \\
17 \\
18 \\
19 \\
20 \\
21 \\
22 \\
23 \\
24 \\
25 \\
26 \\
27 \\
28 \\
29 \\
30 \\
31 \\
32 \\
33 \\
34 \\
35 \\
36 \\
37 \\
38 \\
39 \\
40 \\
41 \\
42 \\
43 \\
44\end{array}$ & $\begin{array}{r}9474.37 \\
73.69 \\
72.89 \\
71.88 \\
71.14 \\
70.32 \\
69.40 \\
68.60 \\
67.74 \\
66.99 \\
66.04 \\
65.19 \\
64.34 \\
63.55 \\
62.64\end{array}$ & $\begin{array}{r}9497.53 \\
96.92 \\
95.96 \\
95.14 \\
94.22 \\
93.32 \\
92.46 \\
91.60 \\
90.73 \\
89.91 \\
89.02 \\
88.11 \\
87.36 \\
86.39 \\
85.62 \\
84.76 \\
83.77 \\
82.99 \\
82.11 \\
7.7 \\
80.08 \\
79.33 \\
78.44 \\
77.47 \\
76.59\end{array}$ & $\begin{array}{c}9514.03 \\
13.24 \\
12.50 \\
- \\
10.04 \\
09.20 \\
08.27 \\
07.41 \\
06.56 \\
05.72 \\
03.97 \\
03.14 \\
02.29 \\
01.36 \\
00.40\end{array}$ & $\begin{array}{r}9545.30 \\
46.02 \\
46.73 \\
47.51\end{array}$ & $\begin{array}{r}9527.74 \\
26.89 \\
26.08 \\
25.23 \\
24.45 \\
23.64 \\
22.03 \\
21.15 \\
20.31 \\
19.39 \\
18.47 \\
17.57 \\
16.64 \\
15.68 \\
14.79\end{array}$ & $\begin{array}{r}9558.21 \\
58.96 \\
59.69 \\
60.40 \\
61.10 \\
61.78 \\
62.48 \\
63.17 \\
63.88 \\
64.61 \\
65.25 \\
65.97 \\
66.65 \\
67.32 \\
68.06 \\
68.76 \\
69.44 \\
70.12 \\
70.91 \\
71.60 \\
72.29 \\
72.95 \\
73.60 \\
74.25 \\
74.92 \\
75.54 \\
76.21 \\
76.80\end{array}$ & $\begin{array}{r}9539.95 \\
39.09 \\
38.35 \\
36.71 \\
35.92 \\
35.02 \\
34.15 \\
33.41 \\
32.50 \\
31.68 \\
30.84 \\
30.02 \\
29.16 \\
28.35 \\
27.30 \\
26.46 \\
25.58 \\
24.74 \\
23.86\end{array}$ & $\begin{array}{l}0 \\
1 \\
2 \\
3 \\
4 \\
5 \\
6 \\
7 \\
8\end{array}$ & $\begin{array}{r}9527.07 \\
485.10 \\
438.00 \\
384.25 \\
326.40\end{array}$ & $\begin{array}{r}\overline{9544.60} \\
537.50 \\
522.92 \\
504.74 \\
480.76 \\
452.62 \\
420.20 \\
384.14\end{array}$ & $\begin{array}{r}9564.82 \\
597.44 \\
622.02 \\
642.93 \\
657.30 \\
666.32\end{array}$ \\
\hline
\end{tabular}

type $A$ bands parallel. For a discussion of pure and hybrid bands and their dependence on molecular symmetry see E. H. Eyster, J. Chem. Phys. 6, 577 (1938). 
TABLE II. The fourth $\mathrm{N}-\mathrm{H}$ harmonic band of hydrazoic acid at $12,412.19 \mathrm{~cm}^{-1}$.

\begin{tabular}{|c|c|c|c|c|c|c|c|c|c|}
\hline$J$ & $Q_{P_{\mathbb{u}(J)}}$ & ${ }^{Q_{R_{3}(J)}}$ & ${ }^{Q} P_{3}(J)$ & $\boldsymbol{e}_{R_{2}(J)}$ & $e_{P_{2}(I)}$ & $Q_{R_{1}(J)}$ & $Q_{P_{1}(J)}$ & $K$ & ${ }^{Q_{O}}{ }_{K}$ \\
\hline $\begin{array}{r}6 \\
7 \\
8 \\
9 \\
10 \\
11^{\circ} \\
12 \\
13 \\
14 \\
15 \\
16 \\
17 \\
18 \\
19 \\
20 \\
21 \\
22 \\
23 \\
24 \\
25 \\
26 \\
27 \\
28 \\
29 \\
30 \\
31 \\
32 \\
33 \\
34 \\
35\end{array}$ & $\begin{array}{r}12,350.64 \\
49.69 \\
48.92 \\
48.02 \\
47.10 \\
46.26 \\
45.47 \\
44.62 \\
43.73 \\
42.86 \\
42.06 \\
41.15 \\
40.42 \\
39.38 \\
38.47 \\
37.56 \\
36.66 \\
35.77 \\
34.88 \\
34.01 \\
33.07 \\
31.99 \\
31.05 \\
30.21 \\
29.35 \\
28.37 \\
27.32\end{array}$ & $\begin{array}{r}12,394.41 \\
95.14 \\
95.77 \\
96.50 \\
97.19 \\
97.93 \\
98.68\end{array}$ & $\begin{array}{r}12,369.13 \\
68.18 \\
67.19 \\
66.34 \\
65.52 \\
64.70 \\
63.83 \\
62.87 \\
61.94 \\
61.03 \\
60.10 \\
59.21 \\
58.34 \\
56.64 \\
55.64 \\
54.69 \\
53.83 \\
52.83\end{array}$ & $\begin{array}{r}12,406.03 \\
06.71 \\
07.54 \\
08.39 \\
09.14 \\
09.87 \\
10.52 \\
11.34 \\
12.04 \\
12.80 \\
13.40 \\
14.19 \\
14.90 \\
15.54 \\
16.25 \\
16.93 \\
17.61 \\
18.29 \\
19.03\end{array}$ & $\begin{array}{r}12,390.03 \\
89.26 \\
88.45 \\
87.57 \\
86.69 \\
85.87 \\
84.91 \\
84.04 \\
83.17 \\
81.45 \\
80.52 \\
79.72 \\
78.87 \\
77.96\end{array}$ & $\begin{array}{r}12,419.73 \\
20.33 \\
21.05 \\
21.77 \\
22.31 \\
23.06 \\
23.97 \\
24.71 \\
25.30 \\
25.97 \\
26.61 \\
27.31\end{array}$ & $\begin{array}{r}12,405.40 \\
04.72 \\
03.99 \\
03.12 \\
02.18 \\
01.34 \\
00.67 \\
\\
\end{array}$ & $\begin{array}{l}1 \\
2 \\
3 \\
4 \\
5 \\
6\end{array}$ & $\begin{array}{r}12,410.60 \\
399.91 \\
382.21 \\
357.69 \\
326.59 \\
289.66\end{array}$ \\
\hline
\end{tabular}

the 250 lines that were measurable in these bands are given in Tables I and II. Missing lines and branches in these tables are ones which are completely obscured by stronger components of the bands; many of the reported lines are blends, and subject to unavoidable errors of measurement for that reason. This is especially true of lines in the third harmonic band, where there are several regions in which the overlapping is so multifold as to produce utter confusion in the fine structure.

\section{Rotational constants}

In the absence of strong perturbations by adjacent vibrational states, the rotational energy of a symmetric rotator may be conveniently written as follows:

$$
F(J, K, v)=\sum_{i, j}^{\prime} X_{i j}{ }^{v}\{J(J+1)\}^{i}\left\{K^{2}\right\}^{j}
$$

The primed summation indicates the omission of the constant term $X_{00} v$, and $v$ is to be considered as the totality of quantum numbers required to specify the vibrational state; in our case it will simply be $v_{1}$, the quantum number associated with the $\mathrm{N}-\mathrm{H}$ stretching frequency. A formula of this type should be expected to describe the majority of the rotational levels of a molecule so nearly symmetric as hydrazoic acid; the leading coefficients will then be related to the effective moments of inertia as follows:

$$
X_{01}{ }^{v}=A_{v}-\frac{1}{2}\left(B_{v}+C_{v}\right) ; \quad X_{10} v=\frac{1}{2}\left(B_{v}+C_{v}\right),
$$

where

$$
\begin{aligned}
& A_{v}=h / 8 \pi^{2} I_{A}{ }^{v} C ; B_{v}=h / 8 \pi^{2} I_{B}{ }^{v} C ; \\
& C_{v}=h / 8 \pi^{2} I_{C}{ }^{v} C ; \quad A_{v} \gg B_{v}>C_{v} .
\end{aligned}
$$

It was found best to evaluate $X_{01}{ }^{0}$ and $X_{02}{ }^{0}$ in the third harmonic band, with the aid of the following relations:

$$
\begin{array}{r}
\Delta F^{\prime \prime}(K) /(2 K+1)=X_{01}^{0}+2 X_{02}^{0} M+\cdots \\
M=\left(K+\frac{1}{2}\right)^{2}+\frac{1}{4} \\
\Delta_{2} F^{\prime \prime}(K) / 4 K=X_{01}{ }^{0}+2 X_{02}{ }^{0} M+\cdots \\
M=K^{2}+1 .
\end{array}
$$

The term differences of (4) are given by:

$$
\begin{aligned}
\Delta F^{\prime \prime}(K) & ={ }^{Q} Q_{K}-{ }^{P} Q_{K+1}={ }^{R} Q_{K}-{ }^{Q} Q_{K+1}, \\
\Delta_{2} F^{\prime \prime}(K) & ={ }^{R} Q_{K-1}-{ }^{P} Q_{K+1} .
\end{aligned}
$$


Eqs. (4) were tested with the experimental combinations, and found to represent the data within experimental error. The constants of the equations were then determined by the method of least squares to be:

$$
X_{01}{ }^{0}=19.946 \mathrm{~cm}^{-1} ; \quad X_{02}{ }^{0}=-0.05616 \mathrm{~cm}^{-1} \text {. }
$$

The dependence of $X_{01}{ }^{v}$ and $X_{02}{ }^{v}$ upon $v$ was established by the determination of $X_{01}{ }^{3}, X_{01}{ }^{4}$, $X_{02}{ }^{3}$, and $X_{02}{ }^{4}$ with the aid of the relation:

$$
\begin{array}{r}
\left({ }^{Q} Q_{1}-{ }^{Q} Q_{K}\right) /\left(K^{2}-1\right)=X_{01}{ }^{\prime \prime}-X_{01}{ }^{\prime} \\
+\left(X_{02}{ }^{\prime \prime}-X_{02}{ }^{\prime}\right) M+\cdots ; \\
M=K^{2}+1 .
\end{array}
$$

This relation was first tested with the observed combinations in both the third and fourth harmonic bands, and found to be well fulfilled; deviations from linearity in $M$ were within the error of observation. The constants were then determined by the method of least squares, and it was found possible to represent the dependence of the terms $X_{01}{ }^{v}$ and $X_{02}{ }^{v}$ upon the vibrational quantum number $v_{1}$ of the $\mathrm{N}-\mathrm{H}$ stretching frequency as :

$X_{01} v_{1}=X_{01}{ }^{0}-\alpha_{01}{ }^{1} v_{1} ; \quad \alpha_{01}{ }^{1}=0.905 \mathrm{~cm}^{-1}$,

$X_{02}{ }^{v_{1}}=X_{02}{ }^{0}-\alpha_{02}{ }^{1} v_{1} ; \quad \alpha_{02}{ }^{1}=-0.000904 \mathrm{~cm}^{-1}$.

It must be realized, of course, that the "lines" used in the above formulas are actually whole branches, and that the appropriate averages over their $J$ structures should appear in Eqs. (4), (5), and (6) to render them precise. This effect will be small, however, since these "lines" are surprisingly sharp. It must also be admitted that the constants $X_{01}{ }^{v}$ and $X_{02}{ }^{v}$ which we have determined in this way may contain small contributions from cross product terms like $X_{11}{ }^{v}$ and $X_{12}{ }^{v}$, but we shall find in the following section that these cross product terms appear to be exceedingly small.

Determination of the constants $X_{10}{ }^{\circ}$ and $X_{20}{ }^{\circ}$ was made by the usual method, with the aid of the expressions :

$$
\begin{gathered}
\Delta_{2} F_{K}{ }^{\prime \prime}(J) / 2(2 J+1)=X_{10}{ }^{0}+K^{2} X_{11}{ }^{0} \\
+2\left(X_{20}{ }^{0}+K^{2} X_{21}{ }^{0}\right) M+\cdots ; \\
M=\left\{\left(J+\frac{1}{2}\right)^{2}+\frac{3}{4}\right\} .
\end{gathered}
$$

The combinations of (8) are the familiar ones:

$$
\Delta_{2} F_{K}{ }^{\prime \prime}(J)={ }^{Q} R_{K}(J-1)-Q P_{K}(J+1) .
$$

When these combinations were evaluated from the three available values of $K$ in the fourth harmonic, their dependence upon $K$ was found to be too small to detect. Hence it has been concluded that the cross terms in the energy expression are too small to be considered in this treatment. The constants $X_{10}{ }^{\circ}$ and $X_{20}{ }^{\circ}$ were then determined by fitting an equation of the form of (8), without the $K$ dependence, to the observed combinations by the method of least squares. The resulting constants, evaluated from the data from the fourth harmonic where overlapping was least serious, were:

$$
X_{10^{0}}=0.3996 \mathrm{~cm}^{-1} ; \quad X_{20}{ }^{0}=-8.1 \times 10^{-6} \mathrm{~cm}^{-1} .
$$

The variation of $X_{10}{ }^{v}$ with vibrational state was best determined by the following relations:

$$
\begin{gathered}
X_{10^{v_{1}}=X_{10}{ }^{0}-\alpha_{10}{ }^{1} v_{1}-\cdots ;} \\
\frac{1}{2}\left\{{ }^{Q} R_{K}(J-1)+Q P_{K}(J)\right\} \\
=f(K)-\alpha_{10}{ }^{1} v_{1} M-\cdots ; \quad M=J^{2} .
\end{gathered}
$$

These combinations were made in the two harmonics, and no systematic deviations from linearity in $M$ could be detected. The average value of the constant $\alpha_{10}{ }^{1}$ was $0.000610 \mathrm{~cm}^{-1}$. The change in the small quantity $X_{20}{ }^{v}$ with vibrational state was beyond the limit of experimental determination.

The rotational constants may now be collected:

$$
\begin{aligned}
& X_{01}{ }^{v}=19.946-0.905 v_{1}-\cdots \\
& X_{02}{ }^{v}=-0.05616+0.000904 v_{1}+\cdots \\
& X_{10}{ }^{v}=0.3996-0.000610 v_{1}-\cdots \\
& X_{20}{ }^{v}=-0.0000081+\cdots
\end{aligned}
$$

\section{Line intensities}

The relative intensities of the lines comprising the parallel component, and the relative intensities of the lines comprising the perpendicular component of the third harmonic band may now be calculated to the symmetric rotator approximation with the aid of the matrix elements of the electric moment given by Dennison for symmetric rotator wave functions. ${ }^{7}$ In order to determine the intensity ratio of the two separate com-

\footnotetext{
${ }^{7}$ D. M. Dennison, Rev. Mod. Phys. 3, 313 (1931).
} 
ponents, however, one must appeal to the experimental intensities, which are very difficult to measure in this region. Fortunately this task was made easy by the fact that two sharp lines of moderate intensity, ${ }^{Q} Q_{6}$ and ${ }^{P} Q_{3}$, which are close together in the third harmonic and in a region quite free from other lines of appreciable intensity, are found to have equal intensities, both by visual examination of prints of the spectrograms and also by inspection of microphotometer traces of the plates. This useful fact establishes the ratio of the parallel and perpendicular components in the third harmonic. Fig. 1 shows these symmetric rotator intensities for the individual coarse structure lines, and the envelopes of the fine structure intensities. It is interesting to note that the calculated intensities of the ${ }^{P} P,{ }^{R} P,{ }^{P} R$, and ${ }^{R} R$ branches are too small to be represented on the scale of Fig. 1 ; no lines of these branches were found. Because of the inherent difficulties of intensity measurements in this region it is quite impossible to make any quantitative comparison of theoretical and observed intensities; suffice it to say that a summation of the intensities of Fig. 1 produces a band envelope whose similarity to a microphotometer trace of the third harmonic band is amazingly close, except for two exceptional lines which will be mentioned shortly. A similar comparison in the fourth harmonic yields comparable results.

This treatment also enables one to calculate the ratios of the vibrational matrix elements of the parallel and perpendicular components of the electric moment. One finds:

$$
\left(M_{\perp}\right)_{03} /\left(M_{\|}\right)_{03}=0.212 ; \quad\left(M_{\perp}\right)_{04} /\left(M_{\|}\right)_{04} \doteq 0 .
$$

It is of interest to notice that there is no evident relationship between these observed ratios and those which one would calculate on the assumption that the change of electric moment takes place along the $\mathrm{N}-\mathrm{H}$ bond, for such an assumption would predict a ratio of 6.7 , nearly independent of $v_{1}{ }^{8}$

Up to this point, no departures from the

${ }^{8}$ This assumption has been used by Badger and Bauer, J. Chem. Phys. 5, 854 (1937). It is probable that the appearance of the third harmonic of the $\mathrm{N}-\mathrm{H}$ stretching frequency in ethylene imine is a result of the failure of this assumption, rather than an indication of an abnormally small angle between the $\mathrm{N}-\mathrm{H}$ bond and the plane of the ring, as was first supposed; see the reference of footnote 6. symmetric rotator intensities have been remarked; such departures do, however, exist, and are found precisely where they should be expected. The ${ }^{Q} P_{0}$ and ${ }^{Q} R_{0}$ branches, whose higher lines should feel the effects of asymmetry most markedly, are completely submerged by stronger branches. The first line of the ${ }^{Q} Q$ branch, ${ }^{Q} Q_{1}$, is already very sharp and in the correct position. The lines ${ }^{R} Q_{0}$ and ${ }^{P} Q_{1}$, however, both of which obtain their greatest intensity from transitions involving levels with $K=0$ for $J$ values around 16 , are noticeably weak and diffuse, the former merely giving anomalous intensity to two fine structure lines, and the latter being almost indistinguishable. The estimated positions of these lines, however, do not seem to deviate greatly from the symmetric rotator predictions, though they were felt unsuitable to use in determining the rotational constants. The next lines in these branches have become very sharp, and no further deviations from the behavior of a symmetric rotator were found in the third harmonic band. None were observed at all in the fourth harmonic where the perpendicular component is absent.

\section{Vibrational Constants}

The vibrational energy expression for hydrazoic acid will have the form:

$$
\begin{aligned}
G(v)=Y_{1}\left(v_{1}+\frac{1}{2}\right)+\cdots+ & Y_{11}\left(v_{1}+\frac{1}{2}\right)^{2} \\
& +\cdots+\text { cross terms. }
\end{aligned}
$$

Determination of the centers of the third and fourth harmonics of the $\mathrm{N}-\mathrm{H}$ frequency of quantum number $v_{1}$ will make possible the evaluation of the vibrational constants $Y_{1}$ and $Y_{11}$. The band centers are given by:

$$
\begin{aligned}
G_{0}{ }^{v}=G(v)-C(0)= & { }^{Q} Q_{K}+K^{2}\left(X_{01}^{0}-X_{01}{ }^{v}\right) \\
& +K^{4}\left(X_{00^{0}}-X_{02}{ }^{v}\right)+\cdots .
\end{aligned}
$$

With the aid of the above expression and the values of the rotational constants already determined, values of $G_{0}{ }^{v}$ were calculated from each of the ${ }^{Q} Q$ lines in the third and fourth harmonic bands, giving as best values:

$$
G_{0}^{3}=9547.31 \mathrm{~cm}^{-1} ; \quad G_{0}^{4}=12,412.19 \mathrm{~cm}^{-1} .
$$

From these the vibrational constants are found 


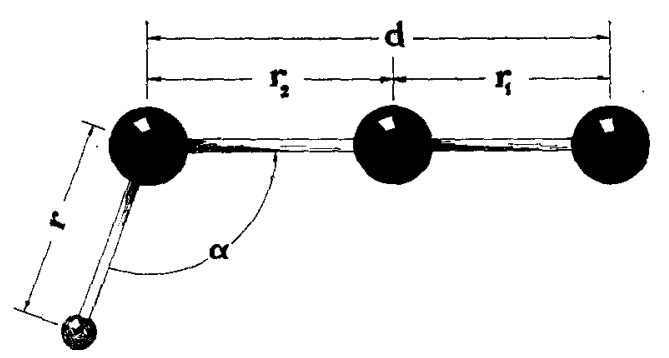

FIG. 2. The assumed model of the hydrazoic acid molecule: the size parameters have the relative values given by this investigation.

to be:

$$
Y_{1}=3497.32 \mathrm{~cm}^{-1} ; \quad Y_{11}=-78.72 \mathrm{~cm}^{-1} .
$$

This information permits the center of the fundamental band to be calculated as 3339.88 $\mathrm{cm}^{-1}$. Davies gives $3336.2 \mathrm{~cm}^{-1}$ as the apparent center of the parallel structure of the fundamental, ${ }^{9}$ and incomplete results in this laboratory give $3335.6 \mathrm{~cm}^{-1}$. Since the apparent minimum of the parallel component will be found at a frequency somewhat lower than that of the actual band center, this agreement seems adequate, and constitutes the justification for the vibrational assignments made for the photographic bands.

\section{Structure of the Molecule}

In order to interpret the rotational constants $X_{01}{ }^{\circ}$ and $X_{10}{ }^{\circ}$ in terms of effective moments of inertia of the ground state of the molecule, it is necessary not only to assume the planar structure, but also to assume explicitly that $I_{A}{ }^{0}+I_{B}{ }^{0}$ $=I_{C}{ }^{0}$; the latter assumption will of course be only approximately true since the $I^{0}$ 's are effective moments of inertia, but for the present purpose this is one of the least important errors. With this assumption and Eqs. (2) and (3), we may obtain :

$$
\begin{gathered}
A_{0}=20.346 \mathrm{~cm}^{-1} ; \quad B_{0}=0.4035 \mathrm{~cm}^{-1} ; \\
C_{0}=0.3957 \mathrm{~cm}^{-1} .
\end{gathered}
$$

The effective moments of inertia, expressed in the convenient chemical units, i.e., mass on the chemical atomic weight scale and distance in $A$, are:

$$
I_{A}{ }^{\prime \prime}=0.8286 ; \quad I_{B}{ }^{\prime \prime}=41.78 ; \quad I_{C}{ }^{\prime \prime}=42.61 .
$$

${ }^{9}$ M. M. Davies, Trans. Faraday Soc. 35, 1184 (1939).
The assumed shape of the hydrazoic acid molecule is shown in Fig. 2. Having assumed the planarity of the molecule and the linearity of the azide group-assumptions in which one has great confidence-the molecule still has four structural parameters, which are conveniently chosen as : $r$, the $\mathrm{N}-\mathrm{H}$ distance; $\alpha$, the $\mathrm{H}-\mathrm{N}-\mathrm{N}$ bond angle; $d$, the length of the azide group; and $k=r_{2} / d$, which places the central nitrogen atom. The moments of inertia can define only two of these parameters, so that we must assume values for the other two. The following have been chosen as the most suitable assumptions:

(1) The parameter $r$ has been chosen as $1.012 \mathrm{~A}$, on the basis of the $1.016 \mathrm{~A} \mathrm{~N}-\mathrm{H}$ distance in the ground state of ammonia. It is felt a decrease of $0.004 \mathrm{~A}$ should be made to care for the absence of the $\mathrm{H}-\mathrm{H}$ repulsions in $\mathrm{HN}_{3}$ and for the fact that the nitrogen atom involved in $\mathrm{N}-\mathrm{H}$ bonding in $\mathrm{HN}_{3}$ is partially double-bonded in the azide group.

(2) The parameter $k$ has been chosen as 0.524 . If one assumes the structures<smiles>[N-]=[N+]=N</smiles>

(a)<smiles>[N-]=[N+]=[N-]</smiles>

(b) to be the only ones contributing appreciably to the ground state of hydrazoic acid, then one can make an approximate calculation of the dependence of both $d$ and $k$ upon $x$, the "hybridization ratio," which is a quantity ranging from zero to one which measures the fractional contribution of state $(a)$ to the ground state. ${ }^{10}$ Fig. 3 shows the result of this calculation. Unfortunately we do not know the value of $x$ which will prevail in the actual molecule, but one sees from Fig. 3 that the azide group can achieve greatest compactness, and hence presumably the greatest binding energy by choosing an $x$ of about 0.63 ; the corresponding $k$ is 0.524 .

One may now determine the values of $\alpha$ and $d$ from the moments of inertia and the assumed values for $k$ and $r$. One finds:

$$
\alpha=110^{\circ} 52^{\prime} ; \quad d=2.369 \mathrm{~A} \text {. }
$$

${ }^{10}$ This method is described and applied to a somewhat different case on page 180, L. Pauling, The Nature of the Chemical Bond (Cornell University Press, 1939). 
Though one might expect the value of $d$ to be less accurately given by the curves of Fig. 3 than is $k$, it is interesting to note that the minimum value of $d$ given by the simple theory is $2.371 \mathrm{~A}$. Such close agreement is of course accidental, but fortunately neither $\alpha$ nor $d$ are very sensitive to reasonable changes of $k$. Table III shows a comparison of the parameters of the azide group as determined by this method, as calculated by the simple theory, and as determined in other compounds containing the azide group. The differences between the values obtained by these four methods are gratifyingly small, being within the limits of error of the diffraction and crystal structure determinations. It is consequently felt that the values

$$
\begin{aligned}
\mathrm{N}_{1}-\mathrm{H} & =1.012 \mathrm{~A} \\
\mathrm{~N}_{1}-\mathrm{N}_{2} & =1.241 \mathrm{~A} \quad \Varangle \mathrm{H}-\mathrm{N}_{1}-\mathrm{N}_{3}=1.128 \mathrm{~A} \\
& =110^{\circ} 52^{\prime}
\end{aligned}
$$

constitute a reasonably precise description of the mean positions of the atoms in the ground state of hydrazoic acid. The structure of the azide group is in complete agreement with the structural theory, and hence tells us nothing new in principle. The angle $\alpha$ is of greater interest, since it might seem somewhat smaller than one would expect on the basis of structures $(a)$ and $(b)$ and a hybridization ratio $(a) /(b)=0.63$. It is felt, however, that this is a real effect, and is another

\begin{tabular}{|c|c|c|c|c|}
\hline PARAMETER & $\begin{array}{c}\text { ELECTRON } \\
\text { DIFFRACTION } \\
\mathrm{CH}_{3} \mathrm{~N}_{3}\end{array}$ & $\begin{array}{c}\text { X-RAY } \\
\text { CRYSTAL } \\
\text { STRUCTURE } \\
\text { CrR }_{3} N_{3}\left(N_{3}\right)_{3}\end{array}$ & $\begin{array}{c}\text { PRESENT } \\
\text { STUDY } \\
\text { HN }_{8}\end{array}$ & $\begin{array}{c}\text { SIMPLE } \\
\text { THEORY } \\
-\mathrm{N}_{3}\end{array}$ \\
\hline $\begin{array}{l}k \\
d \\
\alpha\end{array}$ & $\begin{array}{l}0.530 \\
2.34 \\
120^{\circ}\end{array}$ & $\begin{array}{l}0.532 \\
2.37 \\
-\end{array}$ & $\begin{array}{c}2.369 \\
110^{\circ} 52^{\prime}\end{array}$ & $\begin{array}{l}0.524 \\
2.371 \\
-\end{array}$ \\
\hline
\end{tabular}
example of the tendency of the unshared electron

TABLE III. Parameters of the covalent azide group.

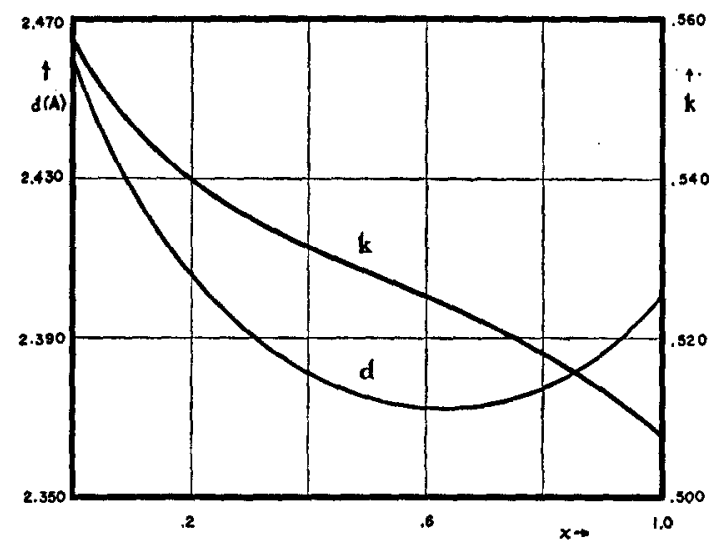

FIG. 3. Dependence of the parameters $d$ and $k=r_{2} / d$ upon the hybridization ratio $x$.

pairs on the nitrogen atom to prefer the $2 s$ orbital, and hence to give to the bonding orbitals somewhat more than the usual $2 p$ character, a procedure which leads to smaller angles.

Finally, this structure makes it easy to understand the large value of the constant $\alpha_{01}{ }^{1}$, which is responsible for the unusual nature of the photographic bands. In fact this constant can be roughly predicted, for with this molecular structure, one sees that $\alpha_{01}{ }^{1}$ should be given approximately by the value of $\alpha_{e}$ for a diatomic molecule with $B_{e}=20.8, \omega_{e}=3497$, and $\omega_{e} x_{e}=78.7$. The Morse approximation, which usually gives too small values for $\alpha_{e}$, then gives $\alpha_{\varepsilon}=0.705$, which is certainly of the right order of magnitude.

In conclusion, the author is pleased to acknowledge his special indebtedness to the National Research Council, and to express his gratitude to Professor Richard M. Badger of the California Institute of Technology, in whose laboratory the experimental part of this investigation was completed, for his helpful advice and for his continued interest in this problem. 